\title{
Alcohol consumption and the incidence of type II diabetes
}

\author{
S G Wannamethee, A G Shaper, I J Perry, K G M M Alberti
}

J Epidemiol Community Health 2002;56:542-548

See end of article for authors' affiliations .....................

Correspondence to: Dr S G Wannamethee, Department of Primary Care and Population

Sciences, Royal Free and University College Medical School, London NW3 2PF, UK; goya@pcps.ucl.ac.uk

Accepted for publication 7 November 2001 alcohol and type II diabetes and the possible Background: This study examines the relation between
mediating effects of HDL-cholesterol and serum insulin.

Methods: Prospective study of 5221 men aged 40-59 years with no history of coronary heart disease, diabetes, or stroke drawn from general practices in 18 British towns.

Results: During the mean follow up of 16.8 years there were 198 incident cases of type II diabetes. Occasional drinkers were the reference group. A non-linear relation was seen between alcohol intake and age adjusted risk of diabetes, with risk lowest in light and moderate drinkers and highest in heavy drinkers (quadratic trend $p=0.03$ ). Further adjustment for body mass index decreased risk in heavy drinkers. After additional adjustment for physical activity, smoking, and (undiagnosed) pre-existing coronary heart disease, only moderate drinkers showed significantly lower risk than occasional drinkers $(\mathrm{RR}=0.6695 \% \mathrm{Cl} 0.44$ to 0.99$)$. Alcohol intake was inversely associated with serum insulin and positively associated with HDL-cholesterol. Adjustment for these factors reduced the "protective" effect in moderate drinkers (adjusted $\mathrm{RR}=0.7395 \% \mathrm{Cl} 0.48$ to 1.10 ) but the quadratic trend remained significant $(p=0.02)$.

Conclusion: There is a non-linear relation between alcohol intake and the risk of type II diabetes. Serum insulin and HDL-cholesterol explained a small amount $(20 \%)$ of the reduction in risk of type II diabetes associated with moderate drinking. The adverse effect of heavy drinking seemed to be partially mediated through its effect on body weight.
T he relation between alcohol intake and risk of type II diabetes has been examined in relatively few prospective studies. ${ }^{1-10}$ Some studies report no association, ${ }^{25}$ while others have suggested that heavy drinking is a risk factor for diabetes. $^{349}$ On the other hand, recent prospective studies suggest that light to moderate drinking may protect against the development of diabetes..$^{6-810}$ This is consistent with observations that low to moderate amounts of alcohol intake increase insulin sensitivity ${ }^{11-14}$ and it is established that insulin resistance and hyperinsulinaemia play an important part in the aetiology of type II diabetes. ${ }^{15}{ }^{16}$ Thus the apparent protective effects of light to moderate drinking may be partially mediated by serum insulin concentrations. An earlier report from the British Regional Heart Study on risk factors for diabetes based on 11.8 years follow up, indicated lower risk in moderate drinkers compared with occasional drinkers even after adjustment for a wide range of factors that included both confounders and possible mediating factors. ${ }^{7}$ Potential confounders were not separated from mediating factors and the role of serum insulin was not examined. This paper examines the relation between alcohol and risk of type II diabetes over a 16.8 year follow up and attempts to determine whether serum insulin and HDL-cholesterol mediate this relation.

\section{METHODS \\ Subjects}

The British Regional Heart Study is a prospective study of cardiovascular disease comprising 7735 men aged 40-59 selected from the age-sex registers of one group general practice in each of 24 towns in England, Wales, and Scotland. The criteria for selecting the town, the general practice, and the subjects as well as the methods of data collection, have been reported. ${ }^{17}$ Research nurses administered a standard questionnaire that included questions on smoking habits, alcohol intake, physical activity, and medical history. The men were asked to recall a doctor's diagnosis of angina, myocardial infarction (heart attack or coronary thrombosis), stroke, diabetes, and several other disorders listed on the questionnaire. Details of the classification of smoking habits, alcohol intake, social class, and physical activity have been reported. ${ }^{17-19}$ The men were classified according to their current smoking status: those who had never smoked, ex-cigarette smokers, and current smokers at four levels $(1-19,20,21-39$, and $\geqslant 40$ cigarettes/day). The men were asked to indicate their usual pattern of physical activity and a total physical score was calculated for each man based on the frequency and type (intensity) of their physical activities. ${ }^{19}$ The men were grouped into six broad categories based on their total score: inactive, occasional, light, moderate, moderately vigorous, and vigorous. Physical measurements including height and weight were made and venous non-fasting blood samples were taken for measurement of biochemical and haematological variables. Aliquots of serum from the men in the 7 th to 24 th towns visited $(n=5663)$ were stored at $-20^{\circ} \mathrm{C}$ and were available for measurement of insulin and triglycerides. Because of the influence that pre-existing cardiovascular disease and diabetes mellitus may have on metabolic risk factor levels and physical activity, men with recall of doctor diagnosis of stroke or coronary heart disease (CHD), known diabetics at screening, and men with asymptomatic hyperglycaemia $(\geqslant 11.1 \mathrm{mmol} / \mathrm{l})$ have been excluded $(\mathrm{n}=441)$. In addition one man did not provide information on alcohol intake and was excluded. After these exclusions, data on insulin were available for a group of 5221 men, the subjects of this study.

\section{Alcohol intake}

Alcohol consumption was recorded at initial screening (Q1) using questions on frequency, quantity, and type. ${ }^{20}$ Initially, eight categories were used: non-drinkers, occasional drinkers (special occasions or 1-2 drinks per month), weekend drinkers (1-2, 3-6 or more than 6 drinks per day), and men drinking 
Table 1 Relative risk of type II diabetes (confidence intervals in parentheses) according to alcohol intake in men free of doctor diagnosed CHD, stroke, or diabetes, with adjustment for age, BMI, lifestyle characteristics, and pre-existing CHD (undiagnosed)

\begin{tabular}{llll}
\hline & \multicolumn{2}{l}{ Adjusted relative risk } & \\
\cline { 2 - 4 } & A & B & C \\
\hline None & $1.12(0.62$ to 2.03$)$ & $1.08(0.60$ to 1.95$)$ & $1.10(0.61$ to 2.00$)$ \\
Occasional & 1.00 & 1.00 & 1.00 \\
Light & $0.75(0.52$ to 1.09$)$ & $0.79(0.54$ to 1.15$)$ & $0.81(0.55$ to 1.20$)$ \\
Moderate & $0.73(0.49$ to 1.08$)$ & $0.70(0.47$ to 1.04$)$ & $0.66(0.44$ to 0.99$)$ \\
Heavy & $1.27(0.81$ to 1.99$)$ & $1.06(0.67$ to 1.66$)$ & $0.96(0.60$ to 1.52$)$ \\
linear trend & $\mathrm{p}=0.42$ & $\mathrm{p}=0.94$ & $\mathrm{p}=0.50$ \\
quadratic trend+ & $\mathrm{p}=0.01$ & $\mathrm{p}=0.03$ & $\mathrm{p}=0.03$ \\
\hline
\end{tabular}

$A=$ age adjusted. $B=$ age $+B M l$ adjusted. $C=$ adjusted for age, $B M I$, smoking, physical activity, social class and pre-existing CHD. Quadratic trend was obtained from a model that included a linear and quadratic term.

daily or on most days (1-2,3-6, or more than 6 drinks per day). One UK unit of alcohol (one drink) is defined as half a pint of beer, a single measure of spirits, or a glass of wine (approximately $8-10 \mathrm{~g}$ alcohol). Twenty five biochemical and haematological measurements on a single blood sample taken at the time the questionnaire was completed by the men in this study indicated that the reported levels of alcohol consumption were valid on a group basis. ${ }^{21}$ The 5221 men were divided into five groups on the basis of their estimated reported weekly intake. ${ }^{18}$

1 Non-drinkers $(n=289)$.

2 Occasional $(<1$ unit/week) $(n=1212)$.

3 Light (1-15 units/week). Weekend 1-2/day, 3-6/day, and daily $1-2(n=1711)$.

4 Moderate (15-42 units/week). Weekend >6/daily and daily 3-6 $(\mathrm{n}=1425)$.

5 Heavy ( $>42$ units/week). Daily $>6$ drinks/day $(n=584)$.

In the analyses examining the relation between alcohol intake and incidence of diabetes, occasional drinkers are used as the baseline group as non-drinkers are a small and heterogeneous group unsuitable for this purpose. ${ }^{22} 23$

\section{Biological measurements}

Body mass index (BMI) calculated as weight/height ${ }^{2}$ was used as an index of relative weight. Obesity is defined as BMI $\geqslant 28.0$ $\mathrm{kg} / \mathrm{m}^{2}$, the top fifth of the distribution in the original 7735 men.

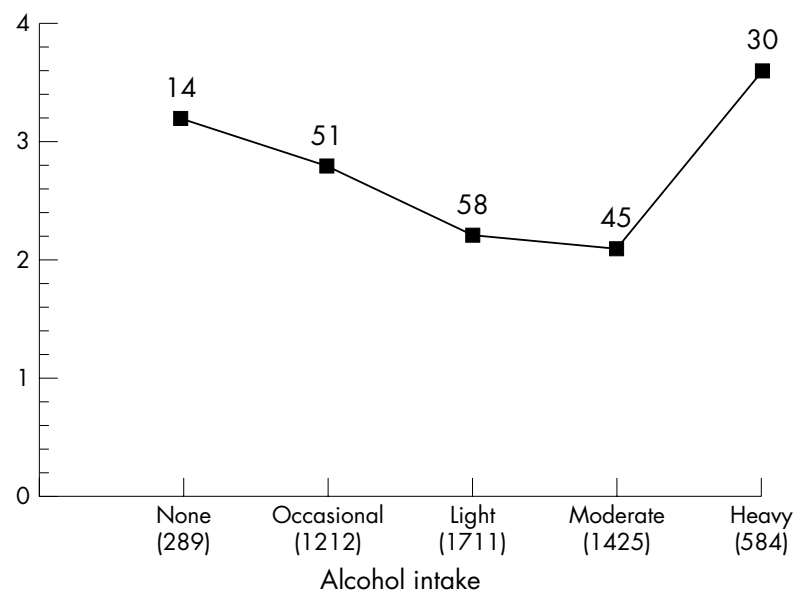

Figure 1 Age adjusted event rate/1000 person years for type II diabetes in 5221 men aged $40-59$ years over an average follow up of 16.8 years according to alcohol intake.
Blood lipids

All the blood samples were obtained in the non-fasting state between 0830 and 1830. Detailed information on blood lipid measurements have been published. ${ }^{24}$ High (raised) HDLcholesterol and high blood glucose are defined as the concentrations in the top fifth of the distribution in the original 7735 men.

\section{Blood glucose}

Glucose was analysed by a glucose oxidase method using an automated analyser (Technicon SMA 12/60). Diurnal variation in glucose levels was modest and no adjustments were made for diurnal variation. ${ }^{25}$

\section{Serum insulin}

Serum insulin concentration was determined by a two site enzyme linked immunoadsorbant assay (ELISA) using commercially available monoclonal antibodies raised against human insulin (Novo Nordisk A/S: Denmark), which do not cross react with pro-insulin. ${ }^{27}$ Analyses were performed in the Department of Medicine, University of Newcastle upon Tyne, UK on non-fasting samples that had been stored at $-20^{\circ} \mathrm{C}$ for between 13 to 15 years. In this laboratory no change in insulin levels was detected in repeat assays of 34 samples, stored at $-20^{\circ} \mathrm{C}$ over an eight year period (mean difference $0.19 \mathrm{mU} / \mathrm{l}$, $\mathrm{p}=0.5$ ). Adjustments were made for the diurnal variation in serum insulin. ${ }^{26}$

High (raised) serum insulin is defined as the concentrations in the top fifth of the distribution in the 5221 men.

Serum insulin was adjusted for time of sampling using a simple mathematical approach that makes no assumptions about the form of the association between these variables and time of sampling. The log transformed data on these variables were adjusted for time of sampling, using the mean level of each variable for each hour in which samples were taken and the grand mean. The calculation for serum insulin was as follows: adjusted log insulin $=$ (unadjusted log insulin-mean log insulin for the hour of sampling) + the grand mean log insulin level. ${ }^{26}$

\section{Pre-existing undiagnosed coronary heart disease}

Men with evidence of CHD (undiagnosed) were defined as those with no recall of a doctor diagnosis of CHD but who had a WHO (Rose) questionnaire response indicating angina or possible myocardial infarction or ECG evidence of definite or possible myocardial ischaemia or myocardial infarction. ${ }^{27}$

\section{Follow up}

All men have been followed up for all cause mortality, cardiovascular morbidity, and development of type II diabetes from 
Table 2 Alcohol intake and unadjusted (SD) and adjusted mean levels of serum HDL-cholesterol (mmol/I) and serum insulin (mU/I) (*geometric mean) in men with no diagnosed CHD, stroke, or diabetes. Adjusted for age, BMI, social class, smoking, physical activity, and pre-existing CHD (undiagnosed)

\begin{tabular}{|c|c|c|c|c|c|c|}
\hline & Non & Occ & Light & Mod & Heavy & test for trend \\
\hline Top fifth \% & 9.3 & 9.7 & 20.0 & 25.4 & 37.0 & \\
\hline Unadjusted HDL-C & 1.06 & 1.07 & 1.14 & 1.20 & 1.28 & \\
\hline & $(0.21)$ & $(0.24)$ & $(0.25)$ & $(0.28)$ & $(0.34)$ & \\
\hline Adjusted HDL-C & 1.04 & 1.05 & $1.11^{\circ}$ & $1.20^{\circ}$ & 1.30 & $p<0.0001$ \\
\hline Top fifth \% & 22.8 & 22.4 & 20.2 & 18.4 & 16.4 & \\
\hline Unadjusted insulin* & 13.2 & 13.2 & 12.4 & 11.2 & 11.4 & \\
\hline (interquartile range) & $(7.7-22.1)$ & $(7.9-21.3)$ & $(7.4-20.0)$ & $(6.9-18.5)$ & $(6.7-18.0)$ & \\
\hline Adjusted insulin* & 12.6 & 12.8 & 11.9 & 11.1 & 10.7 & $p<0.0001$ \\
\hline
\end{tabular}

Top fifth $\%$ = percentage of men in alcohol intake category with concentrations in the top fifth of the distribution in 5221 men.

the initial screening in January 1978-July 1980 to December 1995, a mean period of 16.8 years (range 15.5-18.0 years) and follow up has been achieved for $99 \%$ of the cohort. ${ }^{28}$ Information on death was collected through the established "tagging" procedures provided by the National Health Service registers. Evidence regarding diabetes and non-fatal heart attacks were obtained by reports from general practitioners, by biennial reviews of the patients' notes through to the end of the study period, and from personal questionnaires to surviving subjects at the fifth year and twelfth year after initial examination. A non-fatal heart attack was diagnosed according to WHO criteria. ${ }^{29}$ A diagnosis of diabetes was not accepted on the basis of self completed questionnaire data unless confirmed in the primary care records.

\section{Statistical methods}

The Cox proportional hazards model ${ }^{30}$ was used to assess the effects of alcohol intake on the risk of type II diabetes incidence and risk of CHD. Alcohol intake was fitted as a categorical variable for the five groups (none, occasional, light, moderate, heavy). In some of the analyses, tests for linear trends across the categories of alcohol were assessed by assigning estimated quantitative median values to each level of alcohol intake (that is, none $=0$, occasional $=0.5 /$ week, light $=7 /$ week, moderate $=29 /$ week, heavy $=56 /$ week ) and treating alcohol as a continuous ordinal variable. In addition we considered non-linear trends by adding a quadratic alcohol term (square of the ordinal alcohol variable) to the model. In the adjustment, age, BMI, HDL-cholesterol were fitted as continuous variables. Serum insulin was fitted by fifths of the distribution because of the non-linear relation between serum insulin and diabetes. ${ }^{31}$ Physical activity ( six levels; none, occasional, light, moderate, moderately vigorous, and vigorous), smoking (five levels; never, ex, 1-19, 20, and $\geqslant 21$ cigarettes/ day) and social class (seven groups; I, II, III non-manual, III manual, IV, V, and armed forces) were fitted as categorical variables.

The proportionality assumption for the Cox regression model was tested by including an interaction term for alcohol and follow up time in a time varying analysis. ${ }^{32}$ Direct standardisation was used to obtain age adjusted rates per 1000 person years using the study population as the standard. To determine the possible mediating factors, serum insulin and HDL-cholesterol were fitted in turn to the multivariate model that included age, BMI, smoking, social class, alcohol intake, and pre-existing CHD (undiagnosed). Logistic regression was used to assess the odds of having increased levels of serum insulin and HDL-cholesterol. Analysis of covariance was used to obtain adjusted mean HDL-cholesterol and serum insulin by the five groups of alcohol. Tests for linear trends were obtained by treating alcohol as a continuous ordinal variable.

\section{RESULTS}

During the mean follow up period of 16.8 years there were 198 incident cases of type II diabetes (2.6/1000 person years) in the 5221 men with data on alcohol intake and free of diagnosed CHD, stroke, and diabetes. Figure 1 shows the relation between alcohol intake and age adjusted rates for type II diabetes. A non-linear relation was seen between alcohol intake and diabetes after age adjustment with the lowest rates in moderate drinkers (quadratic trend $p=0.01$ ). Heavy drinkers showed the highest rates followed by non-drinkers. Heavy drinkers showed significantly higher risk than moderate drinkers $(R R=1.71 \mathrm{p}<0.05)$. Adjustment for BMI diminished the increased risk in heavy drinkers (table 1), which now did not differ from occasional or non-drinkers but the quadratic trend remained significant $(p=0.03)$. Further adjustment for potential confounders-that is, social class, physical activity, smoking and CHD (undiagnosed) -reduced the risk in heavy drinkers slightly but the quadratic trend remained significant $(p=0.03)$ and moderate drinkers showed significantly lower risk than occasional drinkers $(p=0.04)$ (table 1).

\section{Alcohol intake and metabolic risk factors}

Table 2 shows the relation between alcohol intake and serum HDL-cholesterol and serum insulin concentrations. These relations have been adjusted for lifestyle characteristics and
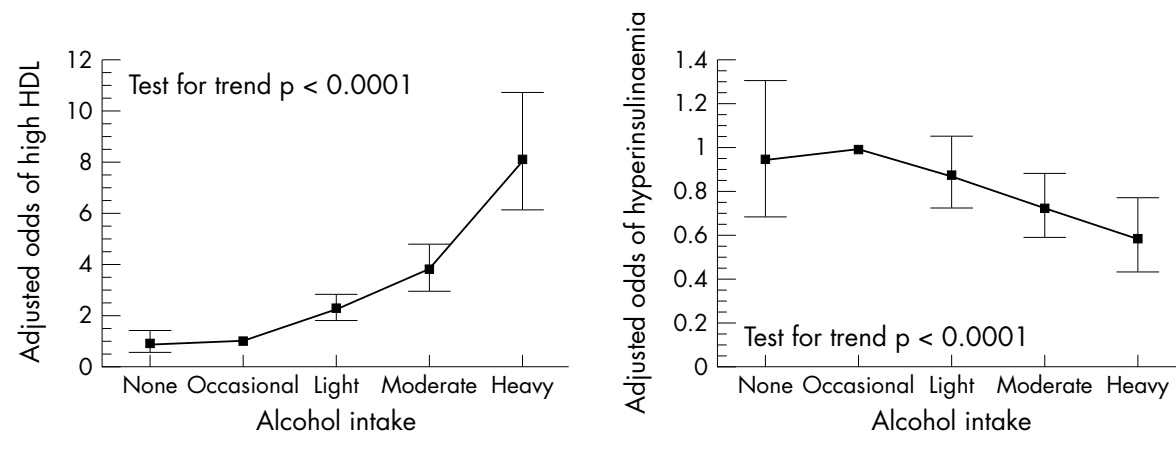

Figure 2 Relative risks (adjusted odds ratios) of high HDL-cholesterol concentration and hyperinsulinaemia and according to alcohol intake. Adjusted for age, BMI, social class, smoking, physical activity, and pre-existing CHD (undiagnosed). 
Table 3 Relative risk of type II diabetes (confidence intervals in parentheses) according to alcohol intake, in men free of doctor diagnosed CHD, stroke, or diabetes adjusting for HDL-cholesterol and serum insulin

\begin{tabular}{|c|c|c|c|c|}
\hline & \multicolumn{4}{|l|}{ Adjusted relative risk } \\
\hline & A & B & c & D \\
\hline \multicolumn{5}{|l|}{ Diabetes } \\
\hline None & $1.10(0.61$ to 2.00$)$ & 1.12 (0.62 to 2.03$)$ & 1.11 (0.60 to 2.01$)$ & 1.12 (0.62 to 2.04$)$ \\
\hline Occ & 1.00 & 1.00 & 1.00 & 1.00 \\
\hline Light & $0.81(0.55$ to 1.20$)$ & $0.84(0.57$ to 1.23$)$ & 0.86 (0.59 to 1.27$)$ & $0.87(0.59$ to 1.29$)$ \\
\hline Mod & $0.66(0.44$ to 0.99$)$ & $0.71(0.47$ to 1.06$)$ & $0.72(0.47$ to 1.10$)$ & $0.74(0.48$ to 1.12 \\
\hline Heavy & $0.96(0.60$ to 1.52$)$ & $1.06(0.66$ to 1.68$)$ & 1.22 (0.76 to 1.97$)$ & 1.24 (0.77 to 1.99$)$ \\
\hline Linear trend & $p=0.50$ & $p=0.81$ & $p=0.75$ & $p=0.71$ \\
\hline quadratic trend* ${ }^{*}$ & $p=0.03$ & $p=0.03$ & $p=0.02$ & $p=0.02$ \\
\hline
\end{tabular}

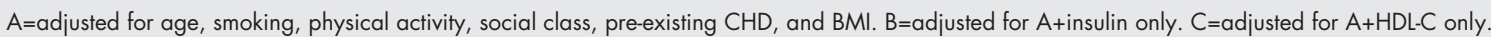
$\mathrm{D}=$ adjusted for $\mathrm{A}+$ +insulin and HDL-C. ${ }^{*}$ Quadratic trend was obtained from a model that included a linear and quadratic term.

pre-existing CHD (undiagnosed). Alcohol intake was positively associated with HDL-cholesterol concentration and inversely associated with insulin concentrations. Figure 2 shows the odds of having raised levels of these factors in the 5221 men after adjustments as in table 2. Heavy drinking was associated with an eightfold increase in having high HDL-cholesterol and a $40 \%$ reduction in risk of having hyperinsulinaemia compared with occasional drinkers.

\section{Alcohol intake, metabolic factors, and risk of type II diabetes}

To assess the possible protective role of HDL-cholesterol and insulin on the relation between alcohol intake and type II diabetes we have examined the effects of additional adjustments for these factors (table 3). Adjustment slightly reduced the protective effects seen in moderate drinkers. Insulin and HDL-cholesterol singly or combined accounted for only about $20 \%$ of the reduction seen and although the reduced risk in moderate drinkers compared with occasional drinkers was no longer statistically significant $(p=0.13)$, the quadratic trend remained significant $(\mathrm{p}=0.04)$ suggesting a non-linear trend between alcohol intake and risk of diabetes.

\section{Alcohol intake, high risk subjects, and diabetes}

We have also examined the relation between alcohol intake and risk of diabetes in men at low and high risk of diabetes (table 4). As the non-drinkers and occasional drinkers showed similar relative risks of diabetes they have been combined to achieve reasonable numbers. Because of the smaller number involved when stratifying we are primarily concerned with whether the associations differ between the levels of these risk factors rather than evaluating the statistical significance within each subgroup. The protective effect of moderate drinking was seen in both men with and without pre-existing CHD (undiagnosed). The protective effect was more evident in men with increased risk of diabetes-that is, heavier men, current smokers, men with low HDL-cholesterol, higher insulin levels, and higher glucose levels. On the other hand, the protective effect was more evident in physically active men. No protective effect was seen in men at low risk-that is, with

Table 4 Incidence rate (per 1000 person years) and adjusted relative risk for diabetes according to risk factors for type II diabetes by alcohol intake. Number of cases/number of men in parentheses). Relative risks adjusted for adjusted for age, smoking, physical activity, social class, pre-existing CHD (undiagnosed), and BMI

\begin{tabular}{|c|c|c|c|c|c|c|}
\hline & & $\begin{array}{l}\text { Rate /1000 } \\
\text { p-y }\end{array}$ & Non/Occ & Light & Mod & Heavy \\
\hline \multicolumn{7}{|c|}{ Pre-existing CHD (undiagnosed) } \\
\hline No & $(141 / 4113)$ & 2.3 & 1.00 & 0.75 & 0.68 & 1.00 \\
\hline Yes & $(57 / 1108)$ & 3.6 & 1.00 & 0.89 & 0.55 & 0.75 \\
\hline \multicolumn{7}{|l|}{ BMI } \\
\hline$<25$ & $(43 / 2427)$ & 1.2 & 1.00 & 0.77 & 0.72 & 0.82 \\
\hline $25-28$ & $(69 / 1814)$ & 2.5 & 1.00 & 0.67 & 0.73 & 1.13 \\
\hline$\geqslant 28$ & $(86 / 980)$ & 6.1 & 1.00 & 0.81 & 0.51 * & 0.89 \\
\hline \multicolumn{7}{|c|}{ Current smoking } \\
\hline No & $(113 / 3015)$ & 2.4 & 1.00 & 0.97 & 0.79 & 0.92 \\
\hline Yes & $(85 / 2200)$ & 2.7 & 1.00 & 0.60 & $0.56^{*}$ & 0.98 \\
\hline \multicolumn{7}{|c|}{ Physical activity } \\
\hline Not active & $(146 / 3243)$ & 3.0 & 1.00 & 0.91 & 0.79 & 0.93 \\
\hline Active & $(50 / 1917)$ & 1.7 & 1.00 & 0.57 & $0.36 *$ & 1.13 \\
\hline \multicolumn{7}{|c|}{ Glucose (tertiles $\mathrm{mmol} / \mathrm{l}$ ) } \\
\hline $1<5.1$ & $(40 / 1848)$ & 1.4 & 1.00 & 0.86 & 1.34 & 1.50 \\
\hline $25.1-$ & $(43 / 1560)$ & 1.8 & 1.00 & 0.60 & 0.54 & 0.97 \\
\hline $35.7-$ & $(115 / 1813)$ & 4.3 & 1.00 & 0.86 & $0.49 *$ & 0.68 \\
\hline \multicolumn{7}{|c|}{ Insulin (tertiles mU/I) } \\
\hline $1<8.6$ & $(27 / 1748)$ & 1.0 & 1.00 & 1.93 & 0.85 & 2.85 \\
\hline $28.6-$ & $(59 / 1735)$ & 2.3 & 1.00 & $0.42 *$ & $0.49 *$ & 0.68 \\
\hline $316.4-$ & $(112 / 1734)$ & 4.4 & 1.00 & 0.92 & 0.81 & 1.00 \\
\hline \multicolumn{7}{|c|}{ HDL-C (tertiles $\mathrm{mmol} / \mathrm{l}$ ) } \\
\hline $1<1.01$ & $(94 / 1725)$ & 3.7 & 1.00 & 0.69 & $0.42 *$ & 1.34 \\
\hline $21.01-$ & $(58 / 1697)$ & 2.3 & 1.00 & 1.08 & 1.25 & 1.26 \\
\hline $31.23-$ & $(42 / 1714)$ & 1.6 & 1.00 & 1.04 & 0.88 & 0.95 \\
\hline
\end{tabular}




\section{Key points}

- Several recent studies suggest a reduced risk of diabetes in light to moderate drinkers compared with non-drinkers.

- In middle aged British men, moderate drinking (15-42 units/week) carries the lowest risk of diabetes.

- The highest risk of diabetes is seen in heavy drinkers (>42 units/week) partly because of increased body weight.

- Alcohol related changes in serum insulin and HDL cholesterol accounted for little of the reduced risk in moderate drinkers.

- The reduced risk of diabetes in moderate drinkers is poorly understood and alcohol is not recommended as a "protec tive" measure.

higher HDL-cholesterol, low insulin levels, or low glucose levels. However, tests for interaction between alcohol and these risk factors on risk of diabetes were not statistically significant.

\section{DISCUSSION}

A non-linear relation was observed between alcohol and incidence of type II diabetes even after adjustment for potential confounders. Risk decreased progressively up to levels of moderate drinking (3-6 drinks/day) and increased in heavy drinkers (daily $>6$ drinks/day). Serum HDL-cholesterol and serum insulin accounted for only a small proportion of the reduced risk seen in moderate drinkers. The protective effect of moderate drinking seemed to be more evident in men at increased risk of diabetes - that is, heavier men, current smokers, men with low HDL-cholesterol, higher insulin levels, and higher glucose levels.

\section{Other studies}

The findings in this study are consistent with several previous prospective studies. In the earlier US Nurses Health Study of 85000 women aged 35-59 years followed up for four years, relative risk of diabetes decreased progressively from 1.0 in non-drinkers to 0.3 in their heaviest drinking category $(\geqslant 15.0 \mathrm{~g} /$ day $) .{ }^{1}$ Additional adjustment for BMI attenuated the protective effect and the possible protective effect of alcohol was considered to be attributable to residual confounding by obesity and its biological consequences. In a later extended follow up study, light drinking (5-10 g/day) was associated with significantly lower risk than in non-drinkers and this was seen in both lean $\left(<25 \mathrm{~kg} / \mathrm{m}^{2}\right)$ and obese $\left(\geqslant 30 \mathrm{~kg} / \mathrm{m}^{2}\right)$ women. ${ }^{33}$ In the US Health Professionals' Study comprising some 42000 men aged 40-75 years followed up for six years, men who drank $30-49.9 \mathrm{~g}$ of alcohol showed a $40 \%$ reduction in risk of diabetes after full adjustment, compared with abstainers and risk increased in the higher intake group $(\geqslant 50$ g/day). ${ }^{6}$ In the Osaka Health Survey of over 6000 Japanese men aged 35-61 years followed up for 10 years, a non-linear relation was seen with the lowest risk in those drinking 29.1-50 g/day ("moderate drinking"). ${ }^{8}$ As in this study, their heavier drinkers ( $\geqslant 50 \mathrm{~g} /$ day) showed the highest risk, but this was attenuated after adjustment that included BMI. Similarly in The Cooper Clinic Study (Texas, USA) of over 8600 men aged 30-79 years followed up for six years a non-linear relation was observed with the lowest risk in those drinking 61.9-122.7 g/week (about 1-2 US drinks/day). . Heavy drinkers ( $>277 \mathrm{~g} /$ week) showed a more than twofold increase in risk compared with these men. In the US Physicians Health Study of 21000 men aged 40-84 years followed up for 12 years, those drinking 2-4 US drinks/week, 5-6 drinks/week, and $\geqslant 1$ drink/day showed significantly lower risk than those who rarely/never drunk with the lowest risk $(R R=0.57)$ in the $\geqslant 1$ drink/day group..$^{10}$ As this was their highest drinking category, it was not possible to assess the effects of heavy drinking in this study. Although the level of drinking associated with reduction in risk of developing type II diabetes varied between these prospective studies, in the majority, light and moderate drinkers have consistently shown lower risk of developing diabetes than heavy drinkers or non-drinkers.

\section{Alcohol and serum insulin concentrations}

An inverse relation was observed between alcohol intake and non-fasting insulin concentrations even after adjustment for potential confounders. The findings suggest that part of the "protective" effect of alcohol on both type II diabetes and CHD may be mediated via effects of alcohol on insulin sensitivity. The finding of lower insulin concentrations in drinkers compared with non-drinkers is consistent with data from previous smaller studies that have examined associations between fasting and post-load insulin and alcohol. ${ }^{11-14}$

\section{Alcohol, HDL-cholesterol, insulin, and type II diabetes} Few studies have examined the relation between alcohol and type II diabetes adjusting for HDL-cholesterol and we are not aware of any prospective study that has examined the influence of insulin on the alcohol-diabetes or CHD relation. In the Cooper Clinic Study the non-linear relation between alcohol intake and diabetes persisted even after adjustment for HDL-cholesterol. ${ }^{9}$ In the present study, HDL-cholesterol and insulin seemed to account for only a small proportion of the reduced risk seen in moderate drinkers in multivariate analysis, although the difference between moderate drinking and occasional drinking (baseline) was no longer statistically significant. The lack of statistical significance may be attributable to the lack of power in categorical analysis when comparing group data. When alcohol was fitted continuously the quadratic trend remained significant after adjustment for insulin and HDL-cholesterol suggesting a non-linear relation between alcohol and diabetes.

\section{Unmeasured and residual confounding}

Studies of this nature will always carry uncertainty arising from unmeasured and residual confounding. For example, we have no measurements of dietary intake or any measures of central obesity, which are both areas of possible confounding. However, the Clinic Cooper study found the non-linear alcohol-diabetes association to be independent of waist circumference and BMI. ${ }^{9}$ The Nurses Health Study found the lower risk associated with light drinking to be independent of dietary factors including trans fatty acids, glycaemic load, fibre, and polyunsaturated fat. ${ }^{33}$ Although we did not separate lifelong teetotallers from ex-drinkers, we excluded a substantial number of people with ill health who may have modified their drinking habits. There is also the possibility of residual confounding arising from imprecise measurement of those variables that have been entered in the adjustment processes.

Bias

It can be argued that more precise measurements of insulin might have attenuated the risk further. The use of non-fasting insulin measurements adjusted for time of sampling has almost certainly increased the amount of random error or "noise" in the data compared with the use of insulin measured under more rigorous conditions. Despite this constraint, previous reports from the British Regional Heart Study have shown increased serum insulin to be associated with increased risk of both type II diabetes and CHD consistent with findings from other insulin-CHD/diabetes studies. ${ }^{2631}$ The associations between non-fasting insulin and cardiovascular risk factors, such as BMI, blood lipids, and blood pressure reported in this cohort are consistent with those reported with fasting and post-load insulin in other studies. ${ }^{34-36}$ Correlation coefficients between insulin and biological risk factors in the BRHS have been shown to be virtually identical to those 
reported from a population based study in eastern Finland in which fasting and two hour plasma insulin values were measured $^{34}$ and the correlation between insulin and these risk factors were similar irrespective of time of sampling. ${ }^{34}{ }^{35}$ This strongly suggests that the use of non-fasting insulin in the present investigation is not associated with systematic measurement error. Furthermore, we have shown recently that insulin and its associated metabolic factors seem to explain a large proportion of the relation between physical activity and type II diabetes. ${ }^{36}$ Thus it is unlikely that the lack of effect of insulin on the alcohol-diabetes and alcohol-CHD relation is simply attributable to the use of non-fasting insulin.

\section{Heavy drinking}

Heavy drinking has been implicated as a risk factor for type II diabetes. $^{39}$ In the majority of prospective studies, heavy drinkers have higher risk than light/moderate drinkers and in many studies heavy drinkers have the highest risk. In the Paris Prospective Study, persons with diabetes had a higher risk of death by cirrhosis, which was strongly associated with alcohol consumption. ${ }^{37}$ In the present study despite the favourable effects of alcohol on HDL-cholesterol and on serum insulin concentrations, heavy drinking was associated with increased risk of diabetes. It has been suggested that the mechanism may be attributable to a direct toxic effect of alcohol on the pancreatic islet cells or may reflect a truncal fat pattern associated with alcohol intake. ${ }^{3}$ In this study, part of the effect of heavy drinking on risk of diabetes seemed to be mediated through its effect on BMI.

\section{Clinical implications}

Despite the consistent association between light to moderate alcohol intake and reduced risk of major coronary heart disease events, virtually all authorities recommend that those who do not drink should not take up drinking and that occasional drinkers should not increase their intake. ${ }^{38}{ }^{39}$ Moreover, as light to moderate alcohol intake does not have the same "protective" effect on non-cardiovascular or all cause mortality as it does on CHD, there is little justification for persuading those who do not drink regularly to do so. ${ }^{40}{ }^{41}$ The same arguments apply to the observation that light to moderate alcohol intake is associated with a reduction in the risk of diabetes. ${ }^{1610}$ Most recent studies have focused on the increased risk of diabetes in men with high alcohol intake and suggested that drinking less might reduce the risk in these subjects. ${ }^{89}$ We need further clinical/physiological studies to explore the complex interrelations between alcohol intake, body weight and obesity, insulin resistance, and lipid metabolism. Present knowledge regarding the modifiable factors involved in the development of diabetes indicate that overweight/obesity, physical activity, and cigarette smoking ${ }^{42}$ should be the key components of any public health programme for the prevention of type II diabetes.

\section{Conclusion}

A non-linear relation was seen between alcohol intake and the incidence of type II diabetes. With occasional drinkers as baseline, risk was lowest in light to moderate drinkers and increased in the heavy drinkers. HDL-cholesterol and serum insulin seemed to explain only some of the reduction in risk of type II diabetes associated with moderate drinking. The adverse effect of heavy drinking on risk of diabetes seemed to be partially mediated through its effect on BMI. Our understanding of the mechanisms underlying the association between light to moderate drinking and the reduced risk of diabetes is limited. The relations between alcohol consumption, obesity, insulin concentrations, and blood lipids need to be explored.

\section{Authors' affiliations}

S G Wannamethee, A G Shaper, Department of Primary Care and Population Sciences, Royal Free and University College Medical School, London, UK

I J Perry, Department of Public Health and Epidemiology, University College Cork, Ireland

K G M M Alberti, Department of Medicine, University of Newcastle upon Tyne, UK

Funding: the British Regional Heart Study is a British Heart Foundation Research Group and receives support from the Department of Health. The views expressed in this publication are those of the authors and not necessarily those of the Department of Health.

Conflicts of interest: none.

\section{REFERENCES}

1 Stampfer MJ, Colditz GA, Willett WC, et al. A prospectve study of moderate alcohol drinking and risk of diabetes in women. Am J Epidemiol 1988; 128:549-58

2 Feskens EJM, Kromhout D. Cardiovascular risk factors and the 25-year incidence of diabetes mellitus in middle-aged men. Am J Epidemiol 1989;130:1101-8.

3 Holbrook TL, Barrett-Connor E, Wingard DL. A prospective population-based study of alcohol and non-insulin-dependent diabetes mellitus. Am J Epidemiol 1990;132:902-9.

4 Balkau B, Randrianjohany A, Papoz L, et al. A prospective study of alcohol use and non-insulin-dependent diabetes mellitus. Am J Epidemiol 1991; 134:1469-70.

5 Hodge AM, Dowse GK, Collins VR Abnormal glucose tolerance and alcohol consumption in three populations at high risk of non-insulin-dependent diabetes mellitus. Am J Epidemiol 1993;137:178-89.

6 Rimm EB, Chan J, Stampfer M, et al. Prospective study of cigarette smoking, alcohol use, and the risk of diabetes in men. BM 1995; 310:555-9.

7 Perry IJ, Wannamethee SG, Walker MK, et al. Prospective study of risk factors for development of non-insulin diabetes in middle-aged British men. BM 1995;310:560-4

8 Tsumara K, Hayashi T, Suetmatsu C, et al. Daily alcohol consumption and the risk of type 2 diabetes in Japanese men. Diabetes Care 1999:22:1432-7.

9 Wei M, Gibbons LW, Mitchell TL, et al. Alcohol intake and incidence of Type 2 diabetes in men. Diabetes Care 2000;23:18-22.

10 Ajani UA, Hennekens CH, Spelsberg A, et al. Alcohol consumption and risk of Type 2 diabetes mellitus among US male physicians. Arch Intern Med 2000; 160: 1025-30.

11 Mayer EJ, Newman B, Quesenberry CP, et al. Alcohol consumption and insulin concentrations. Role of insulin in associations of alcohol intake with high-density lipoprotein cholesterol and triglycerides. Circulation 1993;88:2190-7.

12 Facchini FS, Chen YD, Reaven GM. Light-to-moderate alcohol intake is associated with enhanced insulin sensitivity. Diabetes Care 1994;17:115-19.

13 Kiechl S, Willeit J, Poewe W, et al. Insulin sensitivity and regular alcohol consumption: large prospective, cross sectional population study (Bruneck Study). BM 1996:313:1040-4.

14 Lazarus R, Sparrow D, Weiss ST. Alcohol intake and insulin levels: The Normative Aging Study. Am J Epidemiol 1997;145:909-16.

15 Haffner SM, Stern MP, Mitchell BD, et al. Incidence of type II diabetes in Mexican Americans predicted by fasting insulin and glucose levels, obesity and body fat distribution. Diabetes 1990;39:283-8.

16 Saad MF, Knowler WC, Pettitt DJ, et al. A two step model for development of non-insulin dependent diabetes. Am J Med 1991;90:229-35

17 Shaper AG, Pocock SJ, Walker M, et al. British Regional Heart Study:cardiovascular risk factors in middle-aged men in 24 towns. BM 1981;283:179-86.

18 Shaper AG, Wannamethee G, Walker M. Alcohol and mortality:explaining the U-shaped curve. Lancet 1988;ii: 1268-73.

19 Shaper AG, Wannamethee G. Weatherall R. Physical activity and ishaemic heart disease in middle-aged British men. Br Heart $J$ $1991 ; 66: 384-94$

20 Office of Population Censuses and Surveys. Social Survey Division General Household Survey 1978. London: HMSO, 1980.

21 Shaper AG, Pocock SJ, Ashby D, et al. Biochemical and haematological response to alcohol intake. Ann Clin Biochem 1985; 22:50

22 Wanamethee G, Shaper AG. Men who do not drink: a report from the British Regional Heart Study. Int J Epidemiol 1988;17:307-16.

23 Shaper AG, Wannamethee SG. The J-shaped curve and changes in drinking habit. In: Alcohol and Cardiovascular Diseases Novartis Foundation Symposium No 216. Chichester: Wiley, 1998:173-92.

24 Thelle DS, Shaper AG, Whitehead TP, et al. Blood lipids in middle-aged British men. Br Heart J 1983;49:205-13.

25 Pocock SJ, Ashby D, Shaper AG, et al. Diurnal variations in serum biochemical and haematological measurements. J Clin Pathol 1989;42:172-9. 
26 Perry IJ, Wannamethee SG, Whincup PH, et al. Serum insulin and incident coronary heart disease in middle-aged British men. Am J Epidemiol 1996;144:224-34

27 Cook DG, Shaper AG, Macfarlane PW. Using the WHO (Rose) angina questionnaire in cardiovascular epidemiological studies. Int J Epidemiol 1989:18:607-13

28 Walker $M$, Shaper A G, Lennon L, et al. Twenty year follow-up of a cohort study based in general practices in 24 British towns. J Public Health Med 2000;22:479-85.

29 Rose G, Blackburn H, Gillum RF, et al. Cardiovascular survey methods. 2nd edn. Geneva:WHO, 1982

30 Cox DR. Regression models and life tables (with discussion) J R Stat Soc B 1972;34: 187-220.

31 Perry IJ, Wannamethee SG, Shaper AG, et al. Serum true insulin concentration and the risk of clinical non-insulin dependent diabetes during long term follow-up. Int J Epidemiol 1999;28:735-41.

32 SAS Technical Report P-217. SAS/STAT Software: The PHREG Procedure Vision 6. SAS Institute, 1991

33 Hu FB, Manson JE, Stampfer M, et al. Diet, lifestyle and the risk of type 2 diabetes mellitus in women. N Engl J Med 2001;345:790-7.

34 Wannamethee SG, Shaper AG, Durrington PN, et al. Hypertension, serum insulin, obesity and the metabolic syndrome. J Human Hypertens $1998 ; 12: 735-41$.
35 Mykkanen L, Laakso M, Pyorala K. High plasma insulin level associated with coronary heart disease in the elderly. Am J Epidemiol 1993:137:1190-202.

36 Wannamethee SG, Shaper AG, Alberti KGMM. Physical activity, metabolic factors and the incidence of Type-2 diabetes and coronary heart disease. Arch Intern Med 2000; 160:2108-16.

37 Balkau B, Eschwege E, Ducimetiere P, et al. The high risk of death by alcohol related diseases in subjects diagnosed as diabetic and impaired glucose tolerant: the Paris Prospective Study after 15 years of follow-up. $J$ glucose tolerant: the Paris Prospective
Clin Epidemiol 1991;44:465-74.

38 Shaper AG. Alcohol, the heart and health. Am J Public Health 1993:83:799-801

39 Fagrell B, De Faire U, Bondy S, et al. The effects of light to moderate drinking on cardiovascular diseases. J Intern Med 1999;246:331-40.

40 Wannamethee SG, Shaper AG. Lifelong teetotallers, ex-drinkers and drinkers: mortality and incidence of coronary heart disease events in middle-aged British men. Int J Epidemiol 1997;26:523-31.

41 Hart CL, Davey Smith G, Hole DJ, et al. Alcohol consumption and mortality from all causes, coronary heart disease and stroke: results from a prospective study of Scottish men with 21 years follow-up. BM 1999;318:1725-9.

42 Wannamethee SG, Shaper AG, Perry IJ. Smoking as a modifiable risk factor for type 2 diabetes in middle-aged men. Diabetes Care $2001 ; 9: 1590-5$ 